Hospital Mantuel. Arrizrán.

Cátedra de Pediatria

del Prof. Eugenio Cienfuegos.

\title{
DISOSTOSIS CLEIDO-CRANEANA
}

Por los Dres. JULIO HASBUN y JULIO MENEGHELLO

La disostosis cíleido-craneana es nna afección caracterizada por un trastorno en la osificación del cráneo y de las ciavículas.

Las disostosis cleido-craneanas pueden ser divididas en dos grupos principales:

$11^{\circ}$ Forma familiar y hereditaria, en la cual, la alteración puede estar limitada a un defecto en la osíficacióni de las claviculas (disostosis cleidai) o de las clavículas y huesos membranáceos del cráneo (disostosis cleido-craneana).

2. La más frecuente no es heteditaria ni familiar. pero es congérita y al igual que el grupo anterior puede ser cleidal simple o cleido-craneal.

Esta afección muy rara, fué descrita por Morand (1) en 1766 por primera vez y posteriormente se describieron varios casos, laasta que en 1897 Marie y Sainton (4) proponen el nombre de disostosis cleido-craneana herediraria.

Ultimamente se ha logrado reunir una vasta literatura al respecto, estimándose que el número de casos publicados en la literatura mundial, basta 1939 era de más de 130 casos. según Anspach (2).

En la revisión de la literatura nacional a nuestro alcance, no hemos encontrado ningún caso de disostosis cleidocraneana. Creemos útil, por lo tanto, hacer una breve descripción del cuadro clínico de esta enfermedad, ya que aparentemente nuestra observación constituye la primera en nuestro medio. 
Desde que la alteración caracteristica reside en un defecto fez la osificación de las clavículas, es posible encontrar un número de variedades que puede manifestarse por ausencia total o parcial de ellas, observándose en este último caso unicnes fibrosas entre los fragmentos. Parece que lo más frecuente es lia ausencia de la diáfasis de la clavícula, o la ausencia de la diáfisis de la clavícula y el extremo acromial.

Estos defectos generalmente son bilaterales, pero pueden ser de un solo tado.

Las alteraciones mencionadas constituyen el tipo de discstosis cleidal puro, que es menos frecuente que la disostosis cleido-craneana, donde como su nombre tho indica, además de las alteraciones claviculares, se agregan defectos de los huesos membranáceos del cránec. Así, en estos individuos Mlama la atención desde el nacimiento, la ampilitud de las suturas, las cuales pueden alcanzar hasta cinco centímetros; por otra parte, enormes fontanelas camponen la mayoría del cráneo, en que la fontanela anterior, cemenzando en la raíz de la nariz, se extiende hacia atrás, hasta unirse coto la fontanela posterior.

El cierre de las suturas y fontanelas se hace con lentitud. pero generalmente se logra antes de las 16 años de edad.

Los huresos faciales pueden aparecer como mảs oequeños que lo normal y presentar diversas alteraciones, como prominencia exagerada de ta cresta supraorbitaria, aplanamitnto del piso de la órbita, dando origen a un aparente exoftalmo, paladar ojival y tendencia al prognatísmo. La silla turca es normal.

Con respecto a la dentición, se presenta un atraso e irregularidad en su aparición como en su' reemplazo por los dientes permanentes: estos dientes tienen mala implantación y $z e$ carian tempranamente.

Por parte del sistema muscular se han descrito faltas de desatrollo en la cabeza esternal del esternocleidomastoídeo y en las fibras claviculares del deltoides y trapecio, lo que trae por consecuencia que el hombro pierde su posición normal y cae hacia adelante, simulando una fractura de la clavicula.

Es característico en estos individuos una extraordinaria movilidad de los hombros que les permite aproximarlos por su parte anterior; pueden aproximar y hasta contactar los bordes espinales de los omóplatos en lia aducción posterior forzada de los hombros. 
Junto con el cuadro descrito se han observado asociaciones con otras anomalías, tales como cifosis, escoliosis, lordosis, genu-valgun, coxa-vara, etc.

La mayoría de estos enfermos tiene baja estatụra, men-" talidad, salud y longevidad normales.

El estudio radiológico confirma los hallazgos del examen físico $y$ muchas veces es por este medio que en forma accidental se descubre la afection. La radiolcgía nos permite apreciar la aplasia clavicular total o parcial, uni o bilateral, - las uniones fibrosas entre los fragmentos. El tórax revé!a su forma en embudo con las costillas oblicuamente dirigidas hacia abajo en lugar de su horizontallidad babitual. En el cráneo se comprueba la amplitud de las fontanelas que pueden extenderse desde la raiz de la nariz hasta el occipital. Las suturas se ven anormalmente amplias. En la base del. cráneo hay una detención del crecimiento, especialmente en el sentido transversal y una cifosis de la base del cráneo en oposición a 1'a lordosis de la base que se cibserva en la turricefalia.

Consideraciones etiopatogénicas. - Hasta el momento no se tiene ninguna explicación satisfactoria como causa de esta enfermedad. En el desconcierto etiológice, algunos autrres le han atribuído un papel al raquitismo, la sífilis, a un proceso inflamatorio del cerebro en el nacimiento, y a una retración del amnios con hipertensión del líquido amnió tico (3).

De la revisión de la literatura se desprende que han sido descritos dos tipos fundamentalles de disostosis cleido-craneanas: un primer grupo dende las evidencias hereditarias no ofrecen dudas, y un segundo grupo donde no fué pasible encontrar antecedentes en los familiares. Según Ainspach. ias formas llamadas esporádicas serían hereditarias, pero en las cuales el carácter recesivo es tan poderoso que no se manifieta en más de una generación.

No hay acuerdo entre los autores con respecto a qué clase de hueso es el comprometido, si el membranoso o el cartilaginoso o ambos. El hecho de que muchos de estos indivir duos sean de baja estatura, hace pensar que el bueso formado a base de cartilago pudiera estar afectado. Por otra parte, parece existir actual'mente una tendencia a considerar este cuadro como an trastorno de la osificación general, en que la transformación de membrana a hueso y de cartílago a hueso. está retardada, y no como un proceso estrictamente localizado a las clavículas y, al cráneo. En efecto, se han descrito 
una serie de otras manifestaciones óseas, tales como geni-valgun, escoliosis, vértebras en cuña, ausencia dé osificación de la sínfisis del pubis, alteraciones en las raíces de los dientes permanentes, muchas de las cuales necesitan, para bacerse evidentes, que transcurra un periodo más o menos largo, para que las tracciones musculares $\dot{y}$ el peso del cuerpo ejerzan su influencia,

No hay explicación satisfactoria para el efecto selectivo de la enfermedad scbre las clavículas' y el cráneo.

\section{OBSERVACION CLINICA}

J. C. O. de 49. días de edad, se inscribe en et Pọliclínico del Szanto Obteto de San Miguel el 19 de dieiembre de 1941...Padre de 4Ḅ años de edad. aparentemente sino; sini racciónes de Wassermann y Kabn. Madre de 46 años. aparentemente sana. con reacción de Wassermann negativo $y$ Kahn dudoso en junio de 1940; to presenta ni ha presentado sintomatologia de lúes.

El curso del embarazo fué normal como.asimismo el parto. Ha tenido 5 rmisarazos, de los cuales tress con parto de vérmino y dos abortos espontánzos de tres y dos ruesss.

Tanto los 'padte; coma los tres niños vivos gozan actualmente de buina salud y no prezentan sintomas objetivos ni radiológicos de disostosis.

E. niño nasió de térmirio en sa casa en parto eutórico, el cual fué atendido por una matrona dẹ 'siguro Obrero: no se controló el peso inicial, pato se ¿eja constarcia de su estado de aparente pormaliadad. Fué alimentado al pecho despuci, do las 24 horas da nacer. alimentación que se continúa cada tres horas,

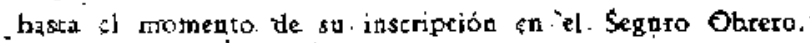

A su ingreso, a los 49 días da edad, se compraéba on niño con buen estado genetal, eutrófico, con un pejo. de $4,400 \mathrm{grs}$. y 54 centímetros de tstatura. Al exámen físico Wama la atcoción la anotmal amplitud de las fontanel te $\mathrm{X}$ suturas En sefecto, se paspaba una amplia depresión longitudina': que, partisndo desdé la raíz de la nariz, pasaba por "la fontanela anterior y se confundía bacia atrás con la fontanela pasterior. Ea el resto del examen no se sescubrió ringuna alteración patológica.

En presencia de esta anormalidad craneana tan marcada, se practica una investigación radiológica de los truesos del cráneo (ver fiģ. 1. y .2) y cuys informe dice: kuezo frontal dividido en dos segmentos por una jaguna mediana que partiendo de la raiz de la nariz, se contigua con ta fontanela ante-

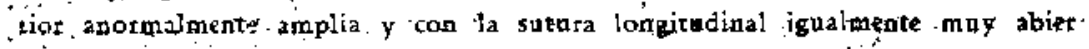
in, para terquizar en la fontanela posterior dé dimensiones érageradas. Una radiografía de tórax (fig. 9) nos muestra un tórạx en forme de enbudo; con las costitis fuertemente oblicuas bacia rbajo. Aplasia clavicular bilateral; - con segspentición de ellas en dos frogmentos, uno de ellos vaido al estemón y et or o stgmento acromial. 


$$
g
$$




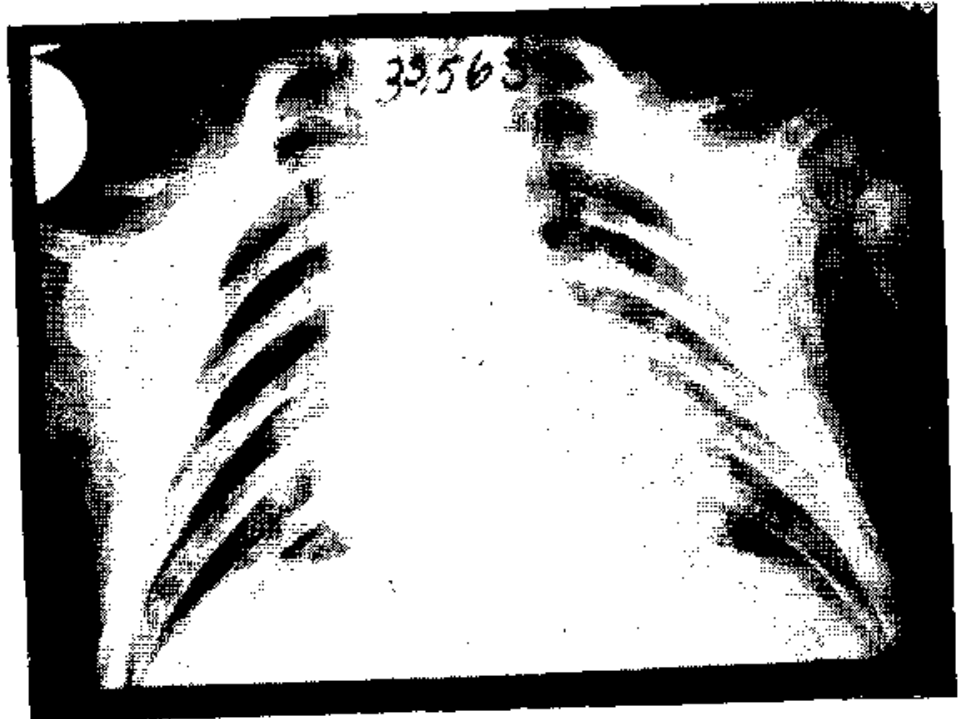

Fìg. 3.

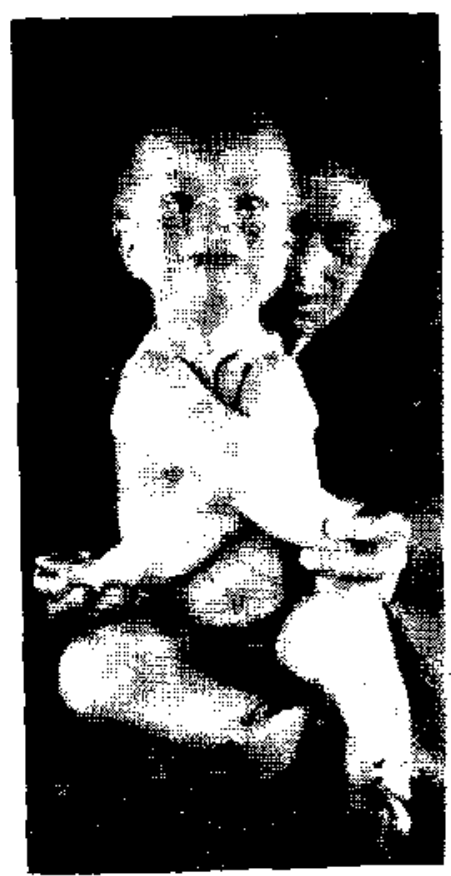

Fis :



Fig. 5. 
Con esta sintomatología rádiolögica, el diagnóstico de disostosis cleidocraneâl no ofrecia diátas y sólo nos restaba proseguir la investigación en los otros mienituros te su familia para su clasificación en su forma esporádica o hereditatia.

Ni el interrogatorio, ni el examen fízico, ni el examen tadiológico practicado los tres hijos y en los padtes, dentostator alteraciones patoiógicas retacionadas con el cuadro que presentamos.

Nuestro enfermo fué observado durante un año, en visitas periódicas al Conseltorio del Seguto Obrerc. En el transcurso de este tiempo fué sometido a un intznso tratamiento a base de vitamina $\mathrm{D}$ y luz vlteaviolecta, "no cbsecvándoor ninguna modificación en la osificación cranesna.

Al año de edad, el niñ̄o peśaba 7.,900 Brs. con dos incisivos medios inferiores y dabz sus primeros pasos apoyado. Ef psiquis desartollado para su edad.

Una fotografía tomada a su edad permite obseryat elatamente la extrat" orfinaria novilidad de los hómbros de esté nifio, pudiendo aproximisrios por cu parte anterior (Figs. 4 y 5 ).

Al año dos meses los padres comarican el fallecimiento brusto del niñon, ignceindose la causa de su muecte.

Resumen. - Se relata la observación de un niño de 49 días de edad que presenta una disostosis décido-craneana. No hubo evidencias de que fuera hereditaria ni familiar.

Se complementa el caso con un estudio de conjunto de la disostosis-cleido-craneana.

\section{Bibliografia.}

1.-MORAND - Citado por Btennemann Prictice of Pediarrics, Vol: IV Eap. 27. págs. 1 , 8 .

2.-ANSPACH, WILLIAM E. y HUEPEL, R, C. - Am. Journal of Diseases of Children. Vol. 58, 787, 1939.

3.-TANSEN, M. Arch. di Ortop. 35: 270,1919 (cit. por Anspach).

4.-MARIE; P. y SAINTON. P. 一 Bụll. et Mèm. Soc. Méd. d'Hop. de. París 14; 706, 1897: Citado por Brennemant Practice of Pediarrics. 